\title{
UNIFORMIDADE DE DISTRIBUIÇÃO NA IRRIGAÇÃO POR GOTEJAMENTO COM ÁGUA RESIDUÁRIA DE PROCESSAMENTO DE MANDIOCA
}

\author{
Eliane Hermes* \\ Marcio Antonio Vilas Boas** \\ Manoel Penachio Gonçalves*** \\ Diego José Gris *** \\ Marcos Araujo Lins ${ }^{* * * * *}$ \\ Jussara Silva Berger ${ }^{* * * * * *}$
}

RESUMO: O objetivo deste estudo consistiu na avaliação de sistemas de irrigação por gotejamento com aplicação de água limpa e água residuária de processamento de mandioca com diferentes concentrações de sólidos suspensos. O experimento foi instalado em uma agroindústria no município de Terra Roxa-PR, sendo montados 12 sistemas. O delineamento experimental foi um fatorial $4 \times 3 \times 2$ considerando-se como fatores: tipo de água aplicada (água limpa e água residuária de processamento de mandioca com 03 concentrações distintas de sólidos suspensos), carga hidráulica (1,5; 2,0 e 2,5 m) e metodologia de coleta (Keller e Karmeli e Denículi), totalizando 24 tratamentos com 30 repetições cada. Previamente foi realizada uma caracterização físico-química da água residuária nas diferentes concentrações de sólidos suspensos para verificar o risco de entupimento das mesmas. A partir dos dados de vazão coletados determinou-se o coeficiente de uniformidade de distribuição (CUD). A concentração de sólidos suspensos presente na água residuária afetou os valores, sendo que com o aumento destes houve pequeno decréscimo na vazão. Os valores de CUD variaram entre duas classificações, sendo dois tratamentos classificados como uma irrigação boa e 22 tratamentos apresentaram valores excelentes.

PALAVRAS-CHAVE: CUD; Qualidade da água; Sólidos suspensos.

Doutora em Engenharia Agrícola, Docente permanente do Programa de Pós-Graduação em Tecnologias de Bioprodutos Agroindustriais da Universidade Federal do Paraná (UFPR), Setor Palotina, Brasil.

** Doutor em Agronomia, Docente permanente do Programa de Pós-Graduação em Engenharia Agrícola - PGEAGRI da UNIOESTE - Campus de Cascavel, Brasil.

*** Graduando em Agronomia da Universidade Federal do Paraná (UFPR), Setor Palotina, Brasil.

**** Graduando em Agronomia (UFSM), Brasil

${ }^{* * * * *}$ Mestre em Engenharia Agrícola pela UNIOESTE, Campus de Cascavel, Brasil.

${ }^{* * * * * *}$ Mestre em Microbiologia Agrícola e do Ambiente pela UFRGS, Brasil. 


\title{
DISTRIBUTION UNIFORMITY ON DRIP IRRIGATION WITH PROCESSED CASSAVA WASTEWATER
}

\begin{abstract}
Irrigation systems by spraying involving clean water and waste water from manioc processing with different concentrations of suspended solids are investigated. The experiment was performed in an agro-industry facility in Terra Roxa, Brazil. Twelve systems were prepared and experiment comprised a 4 × 3 × 2 factorial design. Factors involved type of water (clean water and waste water derived from manioc processing with three different concentrations of suspended solids; hydraulic load $(1.5 ; 2.0 ; 2.5 \mathrm{~m})$ and collection methodology (Keller \& Karmeli \& Denículi), totaling 24 treatments with 30 replications each. The physical and chemical characteristics of waste water at different concentrations of suspended solids were undertaken to verify cogging risks. Discharge data determined the uniformity of distribution coefficient (UDC). Concentration of suspended solids in waste water affected rates. Their increase caused a slight discharge decrease. UDC rates varied between classifications, or rather, two treatments were classified as good irrigation and 22 treatments provided excellent results.
\end{abstract}

KEY WORDS: UDC; Water quality; Suspended solids.

\section{INTRODUÇÃO}

Com a diminuição da disponibilidade de recursos hídricos há a necessidade de que a água seja aplicada primeiramente para duas finalidades: consumo humano e industrialização de alimentos. E com o aumento crescente da população, a quantidade destinada para estas duas utilizações vem se tornando cada vez maior (AL-ABSI et al., 2008; PUIG-BARGUÉS et al., 2010; LIU; HUANG, 2009).

O uso de águas residuárias agroindustriais na agricultura apresenta diversos aspectos positivos, desde o uso potencial de nutrientes presentes mesmo após o seu tratamento, bem como uma aplicação ambientalmente mais adequada destes resíduos que atualmente tem como destinação final em sua maioria os cursos d'água (MEDEIROS et al., 2008; JUCHEN; SUSZEK; VILAS BOAS, 2013; WANG; HUANG, 2008).

A água residuária de processamento de mandioca apresenta-se como um líquido de aspecto leitoso, proveniente das raízes da mandioca, devido a sua 
prensagem para obtenção da fécula ou farinha de mandioca (FIORETTO, 2001). Apresenta-se como importante fonte de nutrientes, já que dispõe de concentrações significativas de nitrogênio, fósforo e potássio, favorecendo o desenvolvimento de culturas.

Para que o seu uso na agricultura não se torne um problema ao meio ambiente, diversos fatores relacionados à sua aplicação precisam ser observados para que a sua exploração seja corretamente manejada. Dentre eles, destacam-se o estudo das necessidades nutricionais das plantas, exigências de solo e qualidade na aplicação do resíduo em toda a área, representada pela uniformidade de distribuição (HERMES, 2013).

$\mathrm{Na}$ avaliação de sistemas de irrigação são utilizadas diferentes metodologias de coleta das vazões aplicadas, como as propostas por Keller e Karmeli (1975) e Denículi et al. (1980). Na primeira, consideram-se vazões amostradas em 16 pontos dentro do sistema, enquanto que na segunda avaliam-se 32 pontos durante o ensaio de irrigação.

A uniformidade na irrigação localizada emprega critérios exigentes em que Pizarro (1996) recomenda o uso do coeficiente de uniformidade de distribuição (CUD). Bralts (1986) classifica seus valores como maior que 90\%, excelente; entre 80 e $90 \%$, bom; 70 e $80 \%$, regular e menor que $70 \%$, ruim.

No caso do uso da irrigação por gotejamento, seu monitoramento é ainda mais importante, pois com o uso de águas de qualidade inferior há possibilidade de problemas ao sistema por dificultar a sua aplicação no campo, principalmente em função do pequeno diâmetro dos emissores e possibilidade de entupimento (AHMED et al., 2006; DAZHUANG et al., 2009; BARROS et al., 2009).

O objetivo deste estudo foi a avaliação de sistemas de irrigação por gotejamento com aplicação de água limpa e água residuária de processamento de mandioca com diferentes concentrações de sólidos suspensos e determinação do CUD.

\section{MATERIAL E MÉTODOS}

A pesquisa foi desenvolvida em uma área plana, sem cobertura vegetal, localizada em uma amidonaria no município de Terra Roxa - PR, cujas coordenadas 
geográficas são $24^{\circ} 11^{\prime} 51.3$ " de latitude Sul e $53^{\circ}$ 59' 09.5" longitude Oeste e altitude de $320 \mathrm{~m}$.

Para a concepção deste projeto foram montados 12 sistemas de irrigação por gotejamento de aproximadamente $66 \mathrm{~m}^{2}(6 \times 11 \mathrm{~m})$ cada, instalados em períodos de tempo distintos. Os sistemas eram constituídos por um tubo gotejador, Streamline 16080 com vazão de $1,49 \mathrm{~L} \mathrm{~h}^{-1}$ a cada $0,30 \mathrm{~m}$ (dados do fabricante), equação

potencial: vazão $=0,1956$ pressão ${ }^{0,4572}$ com coeficiente de determinação $\mathrm{R}^{2}$ de $99,87 \%$. O experimento possuía sete linhas laterais com um total aproximado de 373 gotejadores que equivaleram ao uma vazão total média de aproximadamente $555 \mathrm{~L} \mathrm{~h}^{-1}$.

Com relação ao sistema de filtragem utilizou-se filtro de tela plástica Tavlit de 1" (120 mesh) instalado junto ao reservatório, visando um pré-tratamento dos sólidos, objetivando a minimização de possíveis problemas de entupimento nos sistemas. Cada um dos 12 sistemas possuía um filtro de tela plástica. Pela grande quantidade de material grosseiro retido pelos filtros, a sua limpeza foi realizada a cada três ensaios para todos os tratamentos.

O sistema foi composto de três reservatórios de $1.000 \mathrm{~L}$, que foram elevados a diferentes alturas do chão: 1,5; 2,0 e 2,5 m, aplicadas em épocas diferentes, onde ficaram armazenadas a água limpa e a água residuária de processamento de mandioca.

Foram realizados 30 ensaios por tratamento com espaço de tempo de 1 hora para cada um, sendo o tempo de coleta de 4 minutos para os gotejadores amostrados, mantendo-se as mesmas características de operação dos sistemas para todos os tratamentos.

A pressão foi medida por meio da utilização de dois manômetros digitais ITMPD-15 Instrutemp Modelo 8215 com precisão de $\pm 0,3 \%$ a $\pm 25{ }^{\circ} \mathrm{C}$, sendo esta medida em duplicata a cada ensaio, no início e no final dos sistemas.

A água limpa aplicada nos ensaios de irrigação era oriunda de um poço artesiano instalado próximo à área experimental, sendo utilizada para consumo humano. A água residuária de processamento de mandioca era proveniente do sistema de tratamento da amidonaria. Para a aplicação de diferentes concentrações de sólidos suspensos, após a determinação da concentração presente na água 
residuária da lagoa facultativa foram realizadas duas diluições para a obtenção da água residuária nas três faixas de risco de entupimento descritas por Nakayama; Bucks (1986). A água residuária foi diluída para que se atingissem as concentrações de sólidos suspensos (SS) de inferior a $50 \mathrm{mg} \mathrm{L}^{-1}$, entre 50 e $100 \mathrm{mg} \mathrm{L}^{-1}$ e a terceira faixa de risco foi obtida a partir da água residuária da lagoa facultativa que apresentou concentração superior a $100 \mathrm{mg} \mathrm{L}^{-1}$.

A Tabela 1 traz a caracterização físico-química das águas residuárias de processamento de mandioca utilizadas.

Tabela 1. Caracterização das águas residuárias de processamento de mandioca nas três faixas de risco de entupimento

Água residuária de processamento de mandioca

\begin{tabular}{|c|c|c|c|c|}
\hline Parâmetro & $\begin{array}{c}\text { Concentração } \\
\text { de SS de } 37 \\
\text { mg L }^{-1} \\
\end{array}$ & $\begin{array}{c}\text { Concentração } \\
\text { de SS de } 100 \\
\text { mg L }^{-1}\end{array}$ & $\begin{array}{c}\text { Concentração } \\
\text { de SS de } 466 \\
\text { mg L }^{-1}\end{array}$ & Metodologia \\
\hline Alcalinidade Total $\left(\mathrm{mg} \mathrm{L}^{-1}\right)$ & 177,12 & 202,42 & 607,26 & APHA (2005) \\
\hline Cálcio $\left(\mathrm{mg} \mathrm{L}^{-1}\right)$ & 0,1037 & 0,1203 & 0,2303 & APHA (2005) \\
\hline $\mathrm{CE}\left(\mathrm{dS} \mathrm{m}^{-1}\right)^{*}$ & 1,06 & 0,81 & 3,25 & APHA (2005) \\
\hline $\mathrm{DQO}\left(\mathrm{mg} \mathrm{L}^{-1}\right)$ & $<2,0$ & $<2,0$ & 154 & APHA (2005) \\
\hline Ferro $\left(\mathrm{mg} \mathrm{L}^{-1}\right)$ & 0,0298 & 0,0280 & 0,0814 & APHA (2005) \\
\hline Magnésio $\left(\mathrm{mg} \mathrm{L}^{-1}\right)$ & 0,0065 & 0,0066 & 0,0269 & APHA (2005) \\
\hline Manganês (mg L ${ }^{-1}$ ) & 0,2558 & 0,1002 & 0,3276 & APHA (2005) \\
\hline $\mathrm{pH}$ & 8,0 & 7,7 & 6,8 & APHA (2005) \\
\hline $\operatorname{SDT}\left(\mathrm{mg} \mathrm{L}^{-1}\right)^{* * *}$ & 2199 & 2792 & 7139 & APHA (2005) \\
\hline $\operatorname{SST}\left(\mathrm{mg} \mathrm{L}^{-1}\right)^{* * *}$ & 37 & 100 & 466 & APHA (2005) \\
\hline $\begin{array}{l}\text { Sulfeto de hidrogênio (mg } \\
\mathrm{L}^{-1} \text { ) }\end{array}$ & 5,3 & 4,6 & 12,5 & APHA (2005) \\
\hline Turbidez (UNT) & 6,22 & 9,5 & 43,7 & APHA (2005) \\
\hline
\end{tabular}

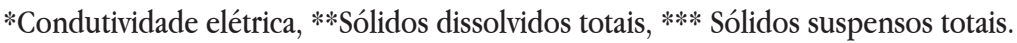

A avaliação dos sistemas consistiu na coleta das vazões aplicadas, considerando-se neste estudo as metodologias propostas por Keller e Karmeli (1975) e Denículi et al. (1980). 
O delineamento experimental foi um fatorial $4 \times 3 \times 2$ : Fator 1 : concentração de sólidos suspensos (SS), fator 2: carga hidráulica e fator 3: metodologia de coleta, com 30 repetições (ensaios) por tratamento, tendo como variável resposta a vazão $\left(\mathrm{L} \mathrm{h}^{-1}\right)$ utilizada na determinação do CUD. A constituição dos tratamentos pode ser visualizada na Tabela 2 .

Tabela 2. Constituição dos tratamentos

\begin{tabular}{|c|c|c|c|c|c|c|c|}
\hline Trat. & $\begin{array}{c}\text { Concentração } \\
\text { de SS } \\
\left(\mathrm{mg} \mathrm{L}^{-1}\right)\end{array}$ & $\begin{array}{c}\text { Carga } \\
\text { hidráulica } \\
\quad(\mathbf{m})\end{array}$ & $\begin{array}{l}\text { Metodologia } \\
\text { de coleta }\end{array}$ & Trat. & $\begin{array}{c}\text { Concentração } \\
\text { de SS } \\
\left(\mathrm{mg} \mathrm{L}^{-1}\right)\end{array}$ & $\begin{array}{c}\text { Carga } \\
\text { hidráulica } \\
\quad(\mathrm{m})\end{array}$ & $\begin{array}{c}\text { Metodologia } \\
\text { de coleta }\end{array}$ \\
\hline $\mathrm{T} 1$ & Água limpa & 1,5 & $\begin{array}{l}\text { Keller e } \\
\text { Karmeli }\end{array}$ & $\mathrm{T} 13$ & ARM (100) & 1,5 & $\begin{array}{l}\text { Keller e } \\
\text { Karmeli }\end{array}$ \\
\hline $\mathrm{T} 2$ & Água limpa & 1,5 & Denículi & T14 & ARM (100) & 1,5 & Denículi \\
\hline $\mathrm{T} 3$ & Água limpa & 2,0 & $\begin{array}{l}\text { Keller e } \\
\text { Karmeli }\end{array}$ & $\mathrm{T} 15$ & ARM (100) & 2,0 & $\begin{array}{l}\text { Keller e } \\
\text { Karmeli }\end{array}$ \\
\hline T4 & Água limpa & 2,0 & Denículi & T16 & ARM (100) & 2,0 & Denículi \\
\hline $\mathrm{T} 5$ & Água limpa & 2,5 & $\begin{array}{l}\text { Keller e } \\
\text { Karmeli }\end{array}$ & $\mathrm{T} 17$ & ARM (100) & 2,5 & $\begin{array}{l}\text { Keller e } \\
\text { Karmeli }\end{array}$ \\
\hline T6 & Água limpa & 2,5 & Denículi & T18 & ARM (100) & 2,5 & Denículi \\
\hline $\mathrm{T} 7$ & ARM (37) & 1,5 & $\begin{array}{l}\text { Keller e } \\
\text { Karmeli }\end{array}$ & T19 & ARM (466) & 1,5 & $\begin{array}{l}\text { Keller e } \\
\text { Karmeli }\end{array}$ \\
\hline $\mathrm{T} 8$ & ARM (37) & 1,5 & Denículi & T20 & ARM (466) & 1,5 & Denículi \\
\hline T9 & ARM (37) & 2,0 & $\begin{array}{l}\text { Keller e } \\
\text { Karmeli }\end{array}$ & $\mathrm{T} 21$ & ARM (466) & 2,0 & $\begin{array}{l}\text { Keller e } \\
\text { Karmeli }\end{array}$ \\
\hline $\mathrm{T} 10$ & ARM (37) & 2,0 & Denículi & $\mathrm{T} 22$ & ARM (466) & 2,0 & Denículi \\
\hline T11 & ARM (37) & 2,5 & $\begin{array}{l}\text { Keller e } \\
\text { Karmeli }\end{array}$ & $\mathrm{T} 23$ & ARM (466) & 2,5 & $\begin{array}{l}\text { Keller e } \\
\text { Karmeli }\end{array}$ \\
\hline $\mathrm{T} 12$ & ARM (37) & 2,5 & Denículi & T24 & ARM (466) & 2,5 & Denículi \\
\hline
\end{tabular}

Trat. - tratamento, ARM - água residuária de processamento de mandioca.

A partir dos dados de vazão coletados foi determinado o coeficiente de uniformidade de distribuição (CUD), sendo este calculado pela Eq. 1.

$$
\operatorname{CUD}(\%)=\frac{100 \times q_{25}}{\bar{q}}
$$


Em que,

$q_{25}$ - vazão média dos $25 \%$ menores valores, $\mathrm{L} \mathrm{h}^{-1}$;

$\overline{\mathrm{q}}$ - vazão média de todas as medições realizadas em $\mathrm{L} \mathrm{h}^{-1}$.

\section{RESULTADOS E DISCUSSÃO}

Os dados relacionados à pressão $(\mathrm{kPa})$ e a vazão $\left(\mathrm{L} \mathrm{h}^{-1}\right)$ obtidos nos 24 tratamentos estão descritos na Tabela 3.

Tabela 3. Pressão média (kPa), vazão média $\left(\mathrm{L} \mathrm{h}^{-1}\right)$ e coeficiente de variação (\%) da vazão para os tratamentos aplicados

\begin{tabular}{cccccccccc}
\hline Trat. & $\mathbf{N}^{*}$ & $\begin{array}{c}\text { Pressão } \\
(\mathbf{k P a})\end{array}$ & $\begin{array}{c}\text { Vazão } \\
\left(\mathbf{L} \mathbf{h}^{-1}\right)\end{array}$ & $\begin{array}{c}\mathbf{C V}^{* *} \\
\mathbf{( \% )}\end{array}$ & Trat. & $\mathbf{N}^{*}$ & $\begin{array}{c}\text { Pressão } \\
(\mathbf{k P a})\end{array}$ & $\begin{array}{c}\text { Vazão } \\
\left(\mathbf{L} \mathbf{h}^{-1}\right)\end{array}$ & $\begin{array}{c}\mathbf{C V}^{* *} \\
(\%)\end{array}$ \\
\hline $\mathrm{T} 1$ & 30 & 16,78 & 0,687 & 8,18 & $\mathrm{~T} 13$ & 30 & 16,25 & 0,730 & 3,45 \\
$\mathrm{~T} 2$ & 30 & 16,78 & 0,691 & 9,29 & $\mathrm{~T} 14$ & 30 & 16,25 & 0,723 & 3,18 \\
$\mathrm{~T} 3$ & 30 & 21,75 & 0,834 & 5,22 & $\mathrm{~T} 15$ & 30 & 21,54 & 0,841 & 3,38 \\
$\mathrm{~T} 4$ & 30 & 21,75 & 0,832 & 5,01 & $\mathrm{~T} 16$ & 30 & 21,54 & 0,840 & 3,40 \\
$\mathrm{~T} 5$ & 30 & 25,84 & 0,835 & 7,96 & $\mathrm{~T} 17$ & 30 & 26,39 & 0,885 & 3,21 \\
$\mathrm{~T} 6$ & 30 & 25,84 & 0,841 & 8,06 & $\mathrm{~T} 18$ & 30 & 26,39 & 0,888 & 2,56 \\
$\mathrm{~T} 7$ & 30 & 18,08 & 0,745 & 3,38 & $\mathrm{~T} 19$ & 30 & 16,66 & 0,708 & 6,26 \\
$\mathrm{~T} 8$ & 30 & 18,08 & 0,734 & 3,32 & $\mathrm{~T} 20$ & 30 & 16,66 & 0,698 & 6,03 \\
$\mathrm{~T} 9$ & 30 & 20,96 & 0,847 & 4,28 & $\mathrm{~T} 21$ & 30 & 21,52 & 0,856 & 2,61 \\
$\mathrm{~T} 10$ & 30 & 20,96 & 0,845 & 4,14 & $\mathrm{~T} 22$ & 30 & 21,52 & 0,859 & 3,51 \\
$\mathrm{~T} 11$ & 30 & 26,55 & 0,896 & 1,98 & $\mathrm{~T} 23$ & 30 & 25,90 & 0,838 & 9,21 \\
$\mathrm{~T} 12$ & 30 & 26,55 & 0,874 & 2,18 & $\mathrm{~T} 24$ & 30 & 25,90 & 0,837 & 9,06 \\
\hline
\end{tabular}

* Número de ensaios; ** coeficiente de variação

A vazão foi influenciada pela pressão aplicada aos sistemas, onde os tratamentos com pressão próxima a $15 \mathrm{kPa}$ apresentaram as menores médias de vazão e os tratamentos com pressão próxima a $25 \mathrm{kPa}$ apresentaram as maiores 
vazões. Ella, Reyes e Yoder (2009) avaliaram o efeito de diferentes pressões na uniformidade de distribuição de água e os ensaios que aplicaram uma pressão de 10 kPa apresentaram médias de vazão menores, em comparação aos demais. Os valores variaram entre 0,500 e $0,550 \mathrm{~L} \mathrm{~h}^{-1}$.

Para todos os tratamentos houve correlação entre as variáveis vazão e pressão, sendo que o aumento de pressão (variável independente) resultou no acréscimo da vazão (variável dependente). A relação entre estas variáveis é expressa pela equação: vazão $=0,464+0,0159$ pressão, com um coeficiente de determinação $\mathrm{R}^{2}=78,1 \%$,

A menor média de vazão $\left(0,687 \mathrm{~L} \mathrm{~h}^{-1}\right)$ foi no tratamento T1 (água limpa, carga hidráulica de 1,5 m e metodologia de coleta de Keller e Karmeli) com mínimo de $0,437 \mathrm{~L} \mathrm{~h}^{-1}$ e máximo de $0,759 \mathrm{~L} \mathrm{~h}^{-1}$. A maior média $\left(0,896 \mathrm{~L} \mathrm{~h}^{-1}\right)$ foi obtida no tratamento T11 (água residuária com concentração de sólidos suspensos de baixo risco $\left(37 \mathrm{mg} \mathrm{L}^{-1}\right)$, carga hidráulica de 2,5 m e metodologia de Keller e Karmeli) com mínimo de $0,859 \mathrm{~L} \mathrm{~h}^{-1}$ e máximo de $0,935 \mathrm{~L} \mathrm{~h}^{-1}$.

Levando-se em conta a concentração de sólidos suspensos $\left(\mathrm{mg} \mathrm{L}^{-1}\right)$ presente na água residuária, os valores de vazão também sofreram influência quando se comparam tratamentos com mesma carga hidráulica e metodologia de coleta variando-se apenas os sólidos em suspensão, em que com o aumento destes na água residuária houve pequeno decréscimo na vazão, com exceção da carga hidráulica de 2,0 $\mathrm{m}$ em ambas as metodologias de coleta.

Ressalta-se que alguns tratamentos com água residuária obtiveram valores de pressão e consequentemente de vazão mais elevados do que os tratamentos com água limpa, o que pode ter ocorrido pelo fato de a água residuária ser levada aos sistemas de irrigação de forma diferente. Para sua condução utilizou-se uma bomba Cv 5 PPM 2900 Amp 15-85 Volts 220-330, instalada junto a lagoa facultativa que ficava a, aproximadamente, $500 \mathrm{~m}$ da área do experimento.

Souza et al. (2005), na utilização de microaspersores com aplicação de água limpa e água residuária de avicultura e bovinocultura, assim como neste trabalho, detectaram alterações nos valores de vazão, em função do risco de entupimento a partir do uso de águas residuárias.

A Tabela 4 apresenta a análise de variância (Anova) para a vazão seguida pelo teste de comparação de médias de Tukey a 5 \% de significância (Tabela 5). 
Tabela 4. Análise de variância da vazão dos gotejadores nos tratamentos aplicados

\begin{tabular}{lccccc}
\hline Fonte de variação & GL & SQ & QM & $\mathbf{F}_{\text {calc }}$ & Prob. \\
\hline Sólidos & 3 & 0,141723 & 0,047241 & $25,150^{* *}$ & 0,0000 \\
Carga & 2 & 3,042769 & 1,521385 & $809,946^{* *}$ & 0,0000 \\
Metodologia & 1 & 0,002714 & 0,002714 & $1,445^{\text {ns }}$ & 0,2297 \\
Sólidos X Carga & 6 & 0,091515 & 0,015253 & $8,120^{* *}$ & 0,0000 \\
Erro & 707 & 1,328013 & 0,001878 & & \\
\hline Total corrigido & 719 & 4,606735 & & & \\
\hline CV (\%) & 5,37 & & & 720 \\
Média geral & 0,8073556 & Número de observações: & \\
\hline
\end{tabular}

ns - não significativo; ** - significativo a 5\% de probabilidade pelo teste $\mathrm{F}$.

Tabela 5. Teste de Tukey para a vazão $\left(\mathrm{L} \mathrm{h}^{-1}\right)$ dos gotejadores nos tratamentos aplicados

\begin{tabular}{cccc|c}
\hline Sólidos suspensos & \multicolumn{5}{c}{ Carga hidráulica (m) } \\
\hline$\left(\mathrm{mg} \mathrm{L}^{-1}\right)$ & 1,5 & 2,0 & 2,5 & Média \\
\hline Água limpa & $0,695 \mathrm{aA}$ & $0,833 \mathrm{aB}$ & $0,838 \mathrm{aB}$ & $0,789 \mathrm{a}$ \\
37 & $0,739 \mathrm{bA}$ & $0,846 \mathrm{abB}$ & $0,885 \mathrm{bC}$ & $0,824 \mathrm{~b}$ \\
100 & $0,726 \mathrm{bA}$ & $0,841 \mathrm{abB}$ & $0,887 \mathrm{bC}$ & $0,818 \mathrm{~b}$ \\
466 & $0,703 \mathrm{aA}$ & $0,857 \mathrm{bC}$ & $0,838 \mathrm{aB}$ & $0,800 \mathrm{a}$ \\
\hline Média & $0,716 \mathrm{~A}$ & $0,844 \mathrm{~B}$ & $0,862 \mathrm{C}$ & 0,807 \\
\hline
\end{tabular}

Médias seguidas pela mesma letra minúscula, na coluna, e maiúscula, na linha, não diferem entre si, pelo teste de Tukey, a $5 \%$ de probabilidade.

Verifica-se com 95\% de confiança que a carga hidráulica e a concentração de sólidos suspensos aplicada afetaram a vazão durante o processo de irrigação, enquanto que a metodologia de coleta não foi significativa ( $p$-valor de 0,2297 ). Notase que a interação entre os dois fatores obteve p-valor inferior a 0,05 , indicando médias estatisticamente diferentes.

O coeficiente de variação foi classificado como baixo (5,37\%), uma vez que foi inferior a 10\%, representando dados homogêneos (GOMES, 2009). O tratamento com água residuária com concentração de sólidos suspensos de risco moderado 
(100 $\mathrm{mg} \mathrm{L}^{-1}$ ) e carga hidráulica de 2,5 m apresentou a maior média de 0,887 $\mathrm{L} \mathrm{h}^{-1}$ e o tratamento com água limpa e carga hidráulica de 1,5 m obteve a menor média de $0,695 \mathrm{~L} \mathrm{~h}^{-1}$. Esta menor média obtida no tratamento com água limpa pode ter sido determinada por este ter sido o primeiro tratamento a ser testado no campo e em função de ajustes necessários no início do experimento isto pode ter afetado negativamente a vazão. Ressalta-se também que o aumento da carga hidráulica proporcionou incremento nos valores de vazão em todas as concentrações de sólidos suspensos testadas.

Os dados relacionados à estatística descritiva do CUD (coeficiente de variação, mínimo e máximo) estão descritos na Tabela 6 .

Tabela 6. Estatística descritiva do CUD para os tratamentos aplicados

(Continua)

\begin{tabular}{cccccc}
\hline Trat. & N* & CUD médio (\%) & CV** (\%) & Mínimo & Máximo \\
\hline T1 & 30 & 91,93 & 2,68 & 87,20 & 95,26 \\
T2 & 30 & 92,87 & 2,30 & 87,67 & 95,68 \\
T3 & 30 & 93,27 & 3,31 & 85,19 & 96,72 \\
T4 & 30 & 94,14 & 2,26 & 88,64 & 96,39 \\
T5 & 30 & 90,97 & 3,60 & 82,19 & 94,40 \\
T6 & 30 & 92,06 & 2,36 & 86,39 & 94,73 \\
T7 & 30 & 92,16 & 2,17 & 89,17 & 96,43 \\
T8 & 30 & 91,03 & 2,14 & 86,59 & 93,99 \\
T9 & 30 & 91,90 & 2,50 & 85,13 & 97,79 \\
T10 & 30 & 91,93 & 2,17 & 86,18 & 95,39 \\
T11 & 30 & 92,96 & 1,79 & 88,79 & 95,67 \\
T12 & 30 & 90,50 & 2,39 & 85,69 & 94,63 \\
T13 & 30 & 91,72 & 3,40 & 81,06 & 95,94 \\
T14 & 30 & 91,65 & 2,28 & 86,08 & 94,11 \\
T15 & 30 & 90,91 & 2,60 & 85,86 & 94,47 \\
T16 & 30 & 90,57 & 2,28 & 85,81 & 95,61 \\
& & & & & \\
\hline
\end{tabular}


(Conclusão)

\begin{tabular}{cccccc}
\hline Trat. & N* & CUD médio (\%) & CV** (\%) $^{*}$ & Mínimo & Máximo \\
\hline T17 & 30 & 87,21 & 3,02 & 81,23 & 92,91 \\
T18 & 30 & 88,31 & 2,20 & 84,35 & 92,48 \\
T19 & 30 & 90,92 & 3,14 & 81,28 & 94,89 \\
T20 & 30 & 90,45 & 2,29 & 84,42 & 92,97 \\
T21 & 30 & 93,23 & 2,92 & 85,62 & 96,91 \\
T22 & 30 & 93,45 & 1,86 & 89,37 & 96,32 \\
T23 & 30 & 92,23 & 2,40 & 85,39 & 97,03 \\
T24 & 30 & 92,37 & 2,12 & 87,56 & 96,16 \\
\hline
\end{tabular}

* Número de ensaios; ** coeficiente de variação

Os valores variaram entre duas classificações, sendo dois tratamentos classificados como uma irrigação boa e 22 tratamentos apresentaram valores excelentes (BRALTS, 1986). A menor média obtida foi para o tratamento T17 (água residuária com concentração de sólidos suspensos de risco moderado (100 mg L $\left.\mathrm{L}^{-1}\right)$, carga hidráulica de 2,5 m e metodologia de coleta de Keller e Karmeli) com valor de $87,21 \%$ com mínimo de $81,23 \%$ e máximo de $92,91 \%$. A maior média foi para 0 tratamento T4 (água limpa com carga hidráulica de 2,0 m e metodologia de coleta de Denículi) tendo o valor médio de $94,14 \%$ com mínimo de $88,64 \%$ e máximo de $96,39 \%$.

Borssoi et al. (2012) avaliaram a uniformidade de aplicação de água e fertilizante, via irrigação por gotejamento, utilizando as metodologias de coleta de Keller e Karmeli e Denículi e determinaram valores de CUD classificados como médio e excelente com médias variando entre 85,84 a 91,65\% para a irrigação e 88,28 a $91,03 \%$ para a fertirrigação. Nota-se a similaridade com o presente trabalho, entre os valores tanto de pressão (12 a $18 \mathrm{kPa}$ ) quanto para o CUD, embora para alguns tratamentos tenham sido obtidas médias superiores de uniformidade, inclusive com o uso de água residuária.

Puig-Bargués et al. (2010) determinaram valores de CUD de 95,1\% em gotejadores com diferentes períodos de limpeza para retirada de partículas 
acumuladas com aplicação de esgoto doméstico tratado. Este resultado apresentase um pouco superior às médias aqui obtidas. Pletsch et al. (2009), em estudo da uniformidade em sistema de irrigação por gotejamento em laboratório como aplicação de esgoto doméstico tratado, determinaram valores de CUD superiores a 98\% para emissores novos, classificados como excelentes, sendo estes mais elevados do que os apresentados neste estudo.

\section{CONSIDERAÇÕES FINAIS}

A carga hidráulica e a concentração de sólidos suspensos aplicadas afetaram a vazão durante o processo de irrigação, enquanto que a metodologia de coleta não foi significativa.

Os valores de CUD variaram entre duas classificações, sendo dois tratamentos classificados como uma irrigação boa e 22 tratamentos apresentaram valores excelentes.

A partir dos resultados obtidos, observa-se que não houve diferença expressiva entre a utilização de água limpa ou de água residuária de processamento de mandioca, demonstrando que esta última aliada a um incremento da carga hidráulica pode ser aplicada com eficiência em um sistema de irrigação por gotejamento.

\section{AGRADECIMENTOS}

Ao CNPq, pelo apoio financeiro no edital MCT/CNPq 14/2010.

A Cooperativa Agroindustrial CVale, pela concessão da área e pelo auxílio na montagem do experimento.

\section{REFERÊNCIAS}

AHMED, B. A.; YAMAMOTO, T.; INOUE, M.; ANYOJI, H. Drip irrigation schedules with saline water for sorghum under greenhouse condition. Trans JSIDRE, v. 244, p. 133-141, 2006. 
AL-ABSI, K. M.; AL-NASIR, F. M.; MAHADEEN, A. Y. Mineral content of three olive cultivars irrigated with treated industrial wastewater. Agricultural Water Management, Amsterdam, v. 96, n. 4, p. 616-626, 2008.

American Public Health Association - APHA; AWWA, WEF. Standard methods for the examination of water and wastewater. 21 ed. Washington: American Public Health Association, 2005.

BARROS, A. C.; COELHO, R. D.; MEDEIROS, P. R. F.; MÉLO, R. F.; BARBOZA JÚNIOR, C. R. A.; DIAS, C. T. Entupimento de gotejadores em função da aplicação de superfosfato simples e ácido nítrico. Engenharia Agrícola, Jaboticabal, v. 29, n. 1, p. 62-71, 2009.

BORSSOI, A. L.; VILAS BOAS, M. A.; REISDORFER, M.; HERNÁNDEZ, R. H.; FOLLADOR, F. A. C. Water application uniformity and fertigation in a dripping irrigation set. Engenharia Agrícola, Jaboticabal, v. 32, n. 4, p. 718-726, 2012.

BRALTS, V. F. Field performance and evaluation. In: NAKAYAMA, F. S.; BUCKS, D. A. Trickle irrigation for crop production. Amsterdam: Elsevier, 1986. p. 216-240.

DAZHUANG, Y.; ZHIHUI, B.; ROWAN, M.; LIKUN, G.; SHUMEI, R.; PEILING, Y. Biofilm structure and its influence on clogging in drip irrigation emitters distributing reclaimed wastewater. Journal of Environmental Sciences, Beijing, v. 21, n. 6, p. 834-841, 2009.

DENICULI, W.; BERNARDO, S.; THIEBAUT, J. T. L. Uniformidade de distribuição de água em condições de campo num sistema de irrigação por gotejamento. Revista Ceres, Viçosa, v. 27, n. 150, p. 155-162, 1980.

ELLA, V. B.; REYES, M. R.; YODER, R. Effect of hydraulic head and slope on water distribution uniformity of low-cost drip irrigation system. Applied Engineering in Agriculture, St. Joseph, v. 25, n. 3, p. 349-356, 2009.

FIORETTO, R. A. Uso direto da manipeuira em fertirrigação. Manejo, uso e tratamento de subprodutos da industrialização da mandioca. São Paulo: Fundação Cargill. (Série: Culturas de Tuberosas Amiláceas Latino Americanas, v. 4), 2001. 
GOMES, F. P. Curso de estatística experimental. 15. ed. Piracicaba: FEALQ, 2009.

HERMES, E. Avaliação da irrigação por gotejamento com aplicação de água residuária de amidonaria. 2013. 134f. Tese (Doutorado em Engenharia Agrícola) Universidade Estadual do Oeste do Paraná, Cascavel, 2013.

JUCHEN, C. R.; SUSZEK, F. L.; VILAS BOAS, M. A. Irrigação por gotejamento para produção de alface fertirrigada com águas residuárias agroindustriais. Irriga, Botucatu, v. 18, n. 1, p. 243-256, 2013.

KELLER, J.; KARMELI, D. Trickle irrigation design. Glendora: Rain Bird Sprinkler Manufacturing, 1975.

LIU, H.; HUANG, G. Laboratory experiment on drip emitter clogging with fresh water and treated sewage effluent. Agricultural Water Management, Amsterdam, v. 96, n. 5 , p. $745-756,2009$.

MEDEIROS, S. S.; SOARES, A. A.; FERREIRA, P. A.; NEVES, J. C. L.; SOUZA, J. A. Utilização de água residuária de origem doméstica na agricultura: Estudo do estado nutricional do cafeeiro. Revista Brasileira de Engenharia Agrícola e Ambiental, v. 12, n. 2, p. 109-115, 2008.

NAKAYAMA, F. S; BUCKS, D. A. Trickle irrigation for crop production: design, operation and management. Amsterdam: Elsevier, 1986.

PIZARRO, C. F. Riegos localizados de alta frecuencia (RLAF) goteo, microaspersión, exudación. 3 ed. Ediciones Mundi Prensa. 1996.

PLETSCH, T. A.; CRUZ, R. L.; MAZZER, H. R.; OLIVEIRA, E. F. Desempenho de gotejadores com uso de esgoto doméstico tratado. Irriga, Botucatu, v. 14, n. 2, p. 243-253, 2009.

PUIG-BARGUÉS, J., ARBAT, G., BARRAGAN, J.; RAMIRAZ de CARTAGENA, F. Hydraulic performance of drip irrigation subunits using WWTP effluents. Agricultural Water Management, Amsterdam, v. 97. p. 883-891, 2010. 
SOUZA, J. A. R.; DENÍCULI, W.; BATISTA, R. O.; VAL, J. C. C.; MATOS, A. T. Suscetibilidade ao entupimento de microaspersores operando com água residuária de bovinocultura. Engenharia na Agricultura, Viçosa, v. 13, n. 3, p. 155-160, 2005. WANG, X.; HUANG, G. Evaluation on the irrigation and fertilization management practices under the application of treated sewage water in Beijing, China. Agricultural Water Management, Amsterdam, v. 95, n. 9, p. 1011-1027, 2008.

Recebido em: 03/02/2016 Aceito em: 29/05/2017 